\title{
Les figures de l'habitant dans les mondes virtuels
}

Jean-François Lucas

\section{OpenEdition}

\section{Journals}

Édition électronique

URL : http://journals.openedition.org/sdj/1353

DOI : $10.4000 /$ sdj. 1353

ISSN : 2269-2657

\section{Éditeur}

Laboratoire EXPERICE - Centre de Recherche Interuniversitaire Expérience Ressources Culturelles Education

\section{Référence électronique}

Jean-François Lucas, «Les figures de l'habitant dans les mondes virtuels », Sciences du jeu [En ligne], 10 | 2018, mis en ligne le 30 octobre 2018, consulté le 19 avril 2019. URL : http:// journals.openedition.org/sdj/1353; DOI : 10.4000/sdj.1353

Ce document a été généré automatiquement le 19 avril 2019.

Tous droits réservés 


\title{
Les figures de l'habitant dans les mondes virtuels
}

\author{
Jean-François Lucas
}

L'auteur tient à remercier Dominique Boullier pour les échanges réguliers qui ont contribué à

façonner ce texte et à clarifier certains concepts et arguments, ainsi que Hovig Ter Minassian pour ses relectures attentives et ses critiques bienveillantes.

1 Un «monde virtuel» est un environnement numérique persistant ${ }^{1}$, multiutilisateur, accessible grâce à internet et dans lequel l'individu interagit avec celui-ci et avec d'autres utilisateurs par le truchement d'un avatar, sa représentation graphique à l'écran.

Les utilisateurs de ces univers, c'est-à-dire les individus qui sont derrière leurs écrans, ou leurs avatars, sont parfois qualifiés d'« habitants » (Damer et alii, 1999; Kaplan, 2007 ; Berry, 2011; Nagy et Koles, 2016). Néanmoins, les caractéristiques permettant de distinguer un utilisateur ou un avatar d'un habitant sont rarement explicitées. Ainsi, en admettant que l'on puisse habiter un monde virtuel, hypothèse que nous questionnerons au fil de notre argumentation tant il semble falloir « un sol, un territoire dans lequel "avoir sa demeure", dans lequel on "vit" " pour habiter (Jankovic, 2003, p. 16), comment différencier un utilisateur qui habite d'un utilisateur qui n'habite pas? Quelles sont les propriétés, les qualités ou les conditions qui permettent de définir qu'un utilisateur, ou son avatar, habite un univers généré par ordinateurs?

Pour Célia Pearce, un monde virtuel est peuplé d'habitants dès lors que ces derniers participent activement à la production, d'une part, d'une culture commune au travers de l'agrégation des rôles et des identités de chacun et, d'autre part, d'une expérience du monde inhérente à l'effet de leurs présences réciproques: "Dans un roman, votre absence n'est pas détectable par les personnages; dans un monde virtuel, elle l'est » (Pearce, 2009, p. 19). Olivier Servais définit quant à lui un habitant du jeu World of Warcraft comme un "praticien intensif» qui considère, "à l'opposé des simples joueurs, le métavers comme une part entière de sa vie sociale et son avatar comme une émulation de soi-même » (Servais, 2012, p. 251). Dans cette perspective, c'est l'intensité de la dimension individuelle de l'expérience de l'utilisateur qui définit un habitant des mondes virtuels. 
Dès lors, comment appréhender le terme « habitant » pour qualifier les utilisateurs ou les avatars de mondes virtuels générés par ordinateurs? D’ailleurs, désigne-t-il l'utilisateur ou son avatar? En outre, faut-il privilégier la dimension collective de l'expérience que vit un individu du fait de l'effet de "présence " généré par la matérialité numérique de son avatar, ou faut-il favoriser sa dimension individuelle, à l'image de l'« intensité » qui peut la caractériser? Il nous semble qu'une troisième voie est possible, à la rencontre de ces deux propositions. Néanmoins, si celle-ci apparaît pertinente, elle se montre également peu originale tant les notions mobilisées de part et d'autre ont déjà fait l'objet de nombreuses recherches. ${ }^{2}$

Dans cet article, nous proposons de traiter précisément du terme d'habitant, et du concept de l'habiter, afin de tenter d'esquisser les contours de cette notion pour comprendre, premièrement, s'il est possible d'habiter un monde virtuel et, deuxièmement, si et comment ce concept peut renouveler les manières de saisir, de décrire et de comprendre des pratiques d'utilisateurs dans ce type d'environnements numériques.

6 Pour répondre à ces objectifs, nous organiserons notre raisonnement en trois temps. D'abord, nous définirons le concept de l'habiter au regard de notre terrain de recherche, les mondes virtuels. Nous expliquerons que pour habiter un monde virtuel, il faut que l'individu ait la sensation d'être «à l'intérieur» de celui-ci. Nous mobiliserons alors le concept d'immersion, puisqu'il permet de décrire un phénomène de projection dans une réalité autre que celle que le corps d'un individu expérimente. Nous décrirons ses principales dimensions et montrerons que l'avatar est une médiation essentielle à la création d'un intérieur au sein d'un monde virtuel, donc à la possibilité de l'habiter.

Dans un second temps, nous traiterons de l'habitabilité des mondes virtuels, c'est-à-dire des propriétés offertes par ces univers pour que les utilisateurs et leurs avatars puissent les habiter. Ce second point part de l'hypothèse que tous les mondes virtuels n'offrent pas les conditions suffisantes aux utilisateurs pour faire l'expérience d'un intérieur. Pour y répondre, nous définirons la notion d'« habitat » à partir des travaux de Georges-Hubert de Radkowski (2002). Son approche nous permettra de préciser que la résidence d'un individu est reconnue comme le lieu où il est présent « de droit » par les " autres ». Alors, nous soulignerons l'importance du code informatique dans la régulation et la nontransgression des droits d'accès et d'action dans les mondes virtuels, puisque pour habiter un lieu dans ce type d'univers numériques, il faut en avoir le droit. Ensuite, nous indiquerons que pour habiter, l'environnement doit "offrir des prises" à l'utilisateur autant que ce dernier doit « avoir prise » sur lui (Bessy et Chateauraynaud, 2014) pour le personnaliser et se l'approprier. Nous présenterons alors différents types de mondes qui proposent aux utilisateurs des latitudes d'actions variées selon ces possibilités d'action.

Dans une dernière partie, enfin, nous explorerons les pratiques des utilisateurs pour comprendre comment ces derniers se saisissent des propriétés des mondes que nous aurons présentées dans la partie précédente afin de pouvoir les habiter. Pour cela, nous présenterons quatre figures de l'habitant dans les mondes virtuels: l'habitantopportuniste, l'habitant-bridé, l'habitant-aménageur et l'habitant-bâtisseur. Au travers de cette typologie, nous mettrons en exergue trois notions, celles de prise, de trace et d'ancrage, autour desquelles l'individu construit son expérience spatiale, donc son rapport au monde et aux autres. Ces propositions nous permettront de fournir une grille de lecture à partir du concept de l'habiter pour observer, décrire et analyser certaines pratiques spatiales d'utilisateurs dans des mondes virtuels. 


\section{Habiter un monde virtuel, c'est être à l'intérieur}

\section{L'utilisateur-habitant des mondes virtuels}

9 À l'image des conceptions de Pearce et de Servais, nous considérons que le terme habitant désigne l'utilisateur, notamment parce que l'avatar n'est pas une entité agissante autonome au sein d'un monde virtuel, mais la représentation, "fût-ce de loin et virtuellement, de véritables êtres humains, des hommes et des femmes qui leur délèguent par procuration une parcelle de légitimité » (Quéau, 1993, p. 73). Or, comment considérer qu'un individu puisse habiter un monde numérique dès lors que sa matérialité, c'est-àdire sa chair, lui empêche d'être "à l'intérieur »? Cette problématique est apparue à de nombreuses reprises lors d'entretiens menés avec des utilisateurs de mondes virtuels, notamment de Second Life, à propos de leurs pratiques de ces univers : ${ }^{3}$ " Habiter ça me semble [...] lourd de sens, c'est comme si j'y étais toujours, mais j'habite dans le monde réel, car mon corps est dans ce monde ", indiquait Charlibix. ${ }^{4}$ Nomaz déclarait quant à lui :» J'ai tendance à dire que je pourrais habiter ce monde. Non pas comme on habite dans le monde réel, car je n'y suis pas réellement, mais il y a quelque chose de similaire. Je préfère dire que je suis un résident ou utilisateur, c'est moins problématique ».

10 Si l'individu qui est derrière son ordinateur ne se détache jamais de son corps charnel, c'est-à-dire de son corps « propre » qui « habite » l'espace physique (Merleau-Ponty, 1945, p.162), de nombreux récits relatent l'impression ou la sensation ressentie par une personne "d'être à l'intérieur » du monde virtuel qu'elle expérimente (Quéau, 1993; Rheingold, 1993 ; Murray, 1997), c'est-à-dire d'être ou d'y être «immergé(e)». Bernard Guelton a défini l'immersion comme reflétant « un puissant sentiment d'absorption du sujet physique et/ou mental produit en situation réelle ou en situation d'appréhension d'une représentation elle-même réaliste ou illusoire. Les perceptions et consciences visuelles, auditives, kinesthésiques constituent autant de modalités différentes et complémentaires à même de produire ce puissant sentiment d'absorption physique, mental et émotionnel » (Guelton, 2014, p. 15).

\section{Modèles et dimensions de l'immersion}

11 Divers modèles de l'immersion ont été établis pour tenter de caractériser les modalités et les manifestations de ce processus, notamment dans les jeux vidéo (Adams, 2004 ; Ermi et Mäyrä, 2005 ; Arsenault, 2006 ; Calleja, 2007). Plusieurs dimensions immersives peuvent ainsi être identifiées.

La première est relative aux qualités sensorielles de l'immersion, qui caractérisent les manières dont les propriétés techniques des dispositifs affectent les sens des individus pour les immerger : la dimension visuelle (l'écran du cinéma ou de l'ordinateur, la trois dimensions (3D), les casques de réalité virtuelle, les CAVE, les simulateurs, etc.), la dimension sonore (la musique, les effets sonores, la spatialisation du son, etc.), la dimension haptique (les contrôleurs avec ou sans retour de force, les combinaisons, etc.), la dimension olfactive ou gustative.

13 La seconde est propre à la jouabilité (gameplay), c'est-à-dire « à la fois à la structure de jeu (game) et à l'attitude ludique (play)», donc "aux modalités d'action laissées au joueur pour agir dans l'œuvre» (Genvo, 2012) et « susciter le (dé)plaisir du joueur, selon ses 
propres représentations et connaissances relatives à l'activité ludique » (Genvo et Bouchardon, 2009, p. 133).

La troisième incarne la dimension narrative de l'immersion puisque l'individu est désormais acteur, et non simple spectateur comme il l'est pour un film ou un livre. Nous parlons d'immersion narrative plutôt que d'immersion fictionnelle (Ermi et Mäyrä, 2005), puisque la narration englobe la fiction: la narration est «l'acte qui produit le récit », le récit étant compris comme "un discours oral ou écrit » dont l'objet est une succession d'événements, réels ou fictifs, c'est-à-dire « une histoire " (Genette, 1972 ; Bouchardon, 2009).

La quatrième correspond à la dimension sociale de l'immersion, qui s'exprime dès lors que l'individu fait l'expérience d'un « engagement partagé » (shared involvement) (Calleja, 2007), c'est-à-dire qu'il « appartient à », « fait partie de », ou encore qu'il est « au sein » d'un collectif (Boullier, 2008).

Enfin, la dimension « désirante », que Dominique Boullier décrit pour le Web et que nous transposons ici aux univers virtuels, permet de caractériser la diversité des mondes virtuels et de leurs usages au travers de la "stimulation constante des désirs » qu'ils procurent, et qui fournit «[...] pour reprendre Sloterdijk [...] à la fois le stress maximum par une sollicitation toujours renouvelée d'objets nouveaux à désirer, et la gâterie maximum, car tout y est disponible» (Boullier, 2008, p. 77).

\section{L'avatar, médiateur central de l'immersion}

17 L'avatar joue un rôle prépondérant dans la réalisation de chacune de ces dimensions immersives. Premièrement, il régit l'immersion perceptive de l'utilisateur, ainsi que les dynamiques de gameplay, puisqu'il incarne ce par quoi les actions et les interactions se réalisent dans le monde virtuel. Deuxièmement, il est un réceptacle pour l'immersion narrative et désirante, puisqu'il accueille et reflète la projection de multiples facettes d'une ou plusieurs identités (Turkle, 1995 ; Auray, 2005 ; Georges 2010), et ce par quoi un individu va vivre de nombreuses histoires (Krueger, 1983; Cooper et Dibbell, 2007; Meadows, 2007 ; Perriault, 2009). Enfin, l'avatar est une condition et un vecteur important de l'immersion sociale, car il signifie, par sa représentation à l'image, la présence de l'utilisateur aux autres membres.

L'avatar est un médiateur central de l'immersion dans les mondes virtuels. Pour autant, s'il contribue à donner à l'utilisateur l'impression que celui-ci est immergé à l'intérieur du monde expérimenté, il ne lui permet pas de faire l'expérience de sa propre intériorité : l'avatar est une coquille vide servant à la fois de réceptacle psychique et de surface malléable que l'on modèle et habille au gré de ses envies et des occasions. Ce n'est donc pas un habitacle, si l'on considère cette notion comme définissant un espace privé, aménageable, personnalisable, que l'individu « vit de l'intérieur " (Fouillé, 2010, p. 25). Il constitue plutôt un « double réceptacle charnel, visuel par le point de vue et matériel par le corps représenté, qui est prêt à accueillir les projections de l'individu dans le monde représenté ", c'est-à-dire une « enveloppe pilotable et plastique » (Lucas et Amato, 2013).

Notons enfin que la qualité de l'incarnation de l'individu dans le monde ne se limite pas à celle de la représentation graphique de l'avatar à l'écran. Elle est constituée d'un réseau de critères et d'affiliations qui regroupent par exemple des »opérateurs ${ }^{5}$ » (les habits, le pseudonyme, etc.), des «qualifiants» (statut, compétences du personnage, etc.), des « 
sociatifs » (les amis) et des « possessifs » (vidéos, photos, terrains possédés, objets produits dans le monde) (Georges, 2010, p. 95).

Ce détour par l'avatar est important pour comprendre l'importance de ce médiateur dans la réalisation de l'immersion, en même temps que son pouvoir relatif à produire un intérieur en tant que tel au sein d'un monde virtuel, soit un habitat pour l'individu.

\section{L'habitabilité des mondes virtuels}

\section{L'habitat-résidence}

Dans son ouvrage Anthropologie de l'habiter, Georges-Hubert de Radkowski (2002) définit l'habitat, c'est-à-dire le lieu d'habitation de l'individu (ou de groupes), dans la complémentarité de ce qu'il nomme "l'habitat-milieu » (ou œkoumène) et «l'habitatcentre » (ou « habitat-résidence » ou résidence). Cette conception est intéressante, car elle conserve une place importante à la résidence, sans restreindre l'habitat à elle seule. Surtout, la résidence définit la zone de « présence de droit » (Radkowski, 2002, p. 44) pour l'individu qui la possède. Le "reste ", c'est-à-dire l'œkoumène, est le lieu de la " présence de fait » (p. 44). Conséquemment, parce qu'elle exprime «le seul lieu où la présence de fait du sujet coïncide avec sa présence de droit ", la résidence constitue "le seul lieu parmi tous les autres qualifié par la présence de l'homme, l'unique endroit où il "demeure", "séjourne" (passe ses jours), "reste", "se tient" (habitare = se habere)» (Radkowski, 2002, p. 44). L'habitat-résidence exprime ainsi la présence (du sujet) « continue quant à l'espace ("partout"), mais discontinue (intermittente) quant au temps » (p. 44). Elle est alors le lieu de la « présence sociale », en tant que lieu où l'individu est « de droit présent aux autres » (Radkowski, 2002, p. 46). C'est donc parce que la résidence « représente le lieu où je suis pour les autres, parce qu'elle est reconnue comme telle [qu'elle] peut devenir mon "chez moi", un espace "privé", considéré par moi comme ma propriété » (p. 46).

\section{Le droit à l'habiter}

La notion de propriété est importante dans les mondes virtuels, puisque le droit régit chaque action de l'utilisateur. Par exemple, dans des univers tels que Second Life et Open Sim, un utilisateur qui possède une parcelle de terrain sera autorisé à créer, modifier et supprimer des objets sur elle, alors qu'il ne pourra pas réaliser ces actions sur un territoire qui ne lui appartient pas; sauf si le propriétaire l'y autorise. Dans le mode « créatif » de Minecraft, à l'inverse, chacun peut agir sur les productions des autres. Mais, si un individu détériore un objet qui ne lui appartient pas (il est alors qualifié de vandale), il risque d'être banni du territoire sur lequel il a réalisé ce méfait. Dans le premier cas, l'application du droit repose sur un système technique qui prémunit le propriétaire terrien de toute altération de son espace. Dans le second, la cohésion des utilisateurs de Minecraft repose d'abord sur une norme, puis sur un système technique qui permet de sanctionner les possibles transgresseurs ${ }^{6}$.

Dans les deux cas, les actions des individus sont régies par les options et les droits du dispositif technique, puisqu'un avatar est autorisé à accéder à un lieu, puis à agir sur lui et à l'occuper, c'est-à-dire à "manifester une emprise » corporelle - par la présence de son avatar - ou matérielle - par ses objets qui encombrent et chargent l'environnement (Sauvage, 2007, p. 75). En ce sens, parce que l'application des droits attribués aux avatars 
est assurée par le code informatique - «Code is law », ne l'oublions pas ! (Lessig, 1999) -, il est impossible pour eux de "squatter » un lieu dans un univers virtuel, si l'on considère qu'un squatteur est une personne sans abri qui s'installe illégalement dans un local vide, dans un immeuble inoccupé ou promis à la démolition. ${ }^{7}$ Ainsi, un avatar qui occupe un lieu y est toujours autorisé, même si le propriétaire de ce dernier n'en est pas avisé ; il peut avoir laissé son territoire ouvert à tous.

\section{De la résidence à son habitation}

Bien qu'un individu puisse occuper la résidence d'un autre, elle reste reconnue par les autres utilisateurs comme le lieu - occupé de droit - de la présence habituelle du propriétaire : «le verbe "habiter" est emprunté du latin habitare, "avoir souvent", comme le précise habitudo qui donne en français "habitude" " (Paquot, 2007, p. 10). Pourtant, occuper habituellement un lieu ne signifie pas habiter, car si « les bâtiments donnent une demeure à l'homme [...] il n'y habite pas, si habiter veut dire seulement que nous occupons un logis » (Heidegger, 1958, 2003, p. 171). Habiter, c'est avoir un lieu à soi que l'on façonne, arrange, aménage et peuple de «ses propres objets chargés de sens (de vécu) et d'histoires familiales ou personnelles" (Leroux, 2008), qui participent à la " continuité temporelle de l'identité» de l'individu (Sansot, 1996, 2004). En somme, "l'action d"'habiter" a une dimension existentielle (Paquot, 2007, p. 13) qui nécessite de s'approprier l'espace investi, de le faire « sien » (Segaud, 2010, p. 72).

Mais, comme nous venons de l'indiquer, pour qu'un individu puisse s'approprier un espace, qu'il en fasse son "habitation », il faut qu'il puisse agir sur celui-ci de différentes manières. ${ }^{8}$ Ces actions doivent donc être "autorisées" par le lieu, puisqu'un lieu " habitable » est avant tout un lieu qui doit » autoriser l'action d'habiter - l'habitation » (Lussault, 2007, p. 38). Ainsi, en plus du droit accordé à l'individu, elles doivent être autorisées en tant que possibles encapsulés dans le dispositif.

\section{Agir dans le monde : latitudes d'action et prises}

Dans son livre GTA IV, l'envers du rêve américain, Olivier Mauco propose le concept de " capacité transformative " pour caractériser « le pouvoir du joueur, au sein du dispositif ludo-fictionnel, d'en modifier l'organisation » (Mauco, 2013, p. 53). Issue des travaux sur la théorie de l'action et la structuration des relations sociales d'Anthony Giddens, cette notion transposée aux jeux vidéo permet de définir « l'ensemble des capacités d'action mécanique, ludique et scénaristique proposées au joueur» (p. 56). Alors, l'action de l'individu « s'opère dans un monde en tension entre ce qui est instancié par la machine et ce qui est actualisable par le joueur » (p. 57). Elle consiste à actualiser les potentiels du monde, afin d'en produire une instance, selon les dispositifs du gameplay (pp. 56-57). De ce point de vue, l'habiter, et son action l'habitation, peuvent être envisagés comme des potentiels encapsulés dans l'architecture technique (le code) du monde virtuel. Autrement dit, soit le monde virtuel, considéré ici comme un ensemble de possibles qui s'offre à l'utilisateur, permet à l'habitation d'être actualisée par celui-ci, soit il ne le permet pas.

27 Cette conception est néanmoins trop unilatérale, puisqu'il ne suffit pas aux utilisateurs de réaliser les possibles offerts par le dispositif technique et ses concepteurs afin d'actualiser une habitation jusqu'alors virtuelle: l'habiter ne se décrète pas, on habite autant qu'on 
est habité, et on peut être habité sans réellement habiter, comme nous allons le voir avec la figure de l'« habitant-opportuniste ». Par ailleurs, si les utilisateurs n'ont pas les mêmes droits dans un univers virtuel, ils n'ont pas non plus les mêmes compétences pour saisir et faire émerger des " prises » (Bessy et Chateauraynaud, 2014) qui leur permettent d'agir sur et avec l'environnement. Pour Bessy et Chateauraynaud, « la notion de prise décrit les relations entre les hommes et les choses en les prenant dans les deux sens : dans le sens d'avoir prise sur, expression qui désigne souvent une ascendance de l'humain (actif, interactif, interrogatif) sur l'objet et l'environnement (inerte, passif, construit) et dans celui de donner prise à, formule qui permet d'accorder aux corps une irréductibilité » (Bessy et Chateauraynaud, 2014, p. 295).

28 Une prise n'est pas donnée a priori, elle est le résultat d'un couplage dynamique entre des "repères", qui sont des "dépôts d'informations " (Bessy et Chateauraynaud, 2014, p. 301) actionnables par les individus, et des "plis ", qui sont de l'ordre de l'action du corps, de l'expérience répétée dans le temps, de la sensation, de l'intuition, de l'expertise, donc relatifs à la subjectivité et aux connaissances de l'individu. La prise établit une relation symétrique entre la capacité d'action de l'individu face aux prises offertes par l'environnement et "des pouvoirs de résistance et de proposition» (Lemieux, 1995, p. 231) que cet environnement offre en retour. La notion de prise est intéressante, car elle ne réduit pas la capacité d'action de l'individu aux saillances ou affordances (Gibson, 1986) offertes par l'environnement. Elle mobilise des savoirs et des représentations, qui sont formalisés par des langages de description (la manière de qualifier un monde virtuel donne déjà des indications sur ses propriétés), un réseau au travers duquel l'objet circule (la connaissance du monde est distribuée dans les autres mondes, dans les forums, dans des vidéos, etc.) ${ }^{9}$, des instruments qui vont permettre d'agir sur le monde (un langage de script dans Second Life par exemple, pour que ces derniers puissent animer les objets qu'ils produisent) et l'expérience sensorielle (en premier lieu la perception visuelle dans notre cas, mais on peut également penser à l'engagement du corps de l'utilisateur de manière générale, de sa posture, du jeu continuel de ses mains sur un clavier, une souris ou encore une manette).

C'est pourquoi, nous disent Bessy et Chateauraynaud, ce n'est pas la quantité de savoir qui différencie un expert d'un profane, mais la manière dont ces deux figures vont être engagées dans le processus dynamique de création de prises : «Le profane, en dépit de son intuition des plis, utilisera plus volontiers des repères communs là où l'expert s'appuiera sur des prises capables de faire la relation entre repères et plis " (Bessy et Chateauraynaud, 2014, p. 304). La notion de prise apparaît pertinente pour différencier des pratiques qui résultent de l'utilisation de repères partagés par tous, et d'autres qui sont le fruit de réinterprétations et d'arrangements grâce à l'expertise dont témoignent certains utilisateurs qui font "plier» le monde selon des formes d'action ou d'interprétation spécifiques. Pour identifier les prises offertes aux avatars à l'intérieur d'un monde virtuel, ou les manières dont ils peuvent en faire émerger de nouvelles, il est donc nécessaire d'étudier autant les modalités structurelles et configurationnelles de l'environnement représenté, que le capital culturel (savoirs, compétences, etc.), voire sensoriel, des utilisateurs. 


\section{Différents types d'espaces « pour soi »}

30 Certains univers numériques, comme ceux des jeux vidéo dits à « monde ouvert » (ou open world), proposent une grande latitude aux utilisateurs (des « joueurs » dans ce cas) dans le choix de leurs déplacements ou encore dans la manière dont ils construisent leur propre récit (autant dans l'ordre des événements que dans leurs natures). Cela leur permet d'actualiser une jouabilité toujours plus singulière, ce qui incite certains à considérer ce type de jeux vidéo comme des espaces à habiter (Beaude, 2012, p. 50 ; Joliveau, 2012, p.66). Nous considérons à l'inverse que ces jeux dits à monde ouvert restent le plus souvent fermés quant aux interactions qu'un utilisateur a avec l'environnement modélisé en trois dimensions, puisqu'il ne peut le plus souvent pas agir sur sa forme et sa structure. Ainsi, nous préférons parler de "systèmes de jeu ouverts", plutôt que de "mondes ouverts ", car les contenus restent déterminés, puisqu'encapsulés dans un ensemble de possibles défini par des concepteurs et actualisable par l'utilisateur. Dans cette perspective, les mondes virtuels de création de contenus (MVCC) (Lucas, 2012), tels que Second Life et Minecraft ${ }^{10}$, offrent une plus grande latitude aux utilisateurs pour agir sur et avec l'espace, puisque ce sont eux qui façonnent les environnements numériques et les usages des univers qu'ils investissent.

31 Nous proposons alors de distinguer :

32 - les univers à système de jeu ouvert, à l'image des opus de la série Grand Theft Auto (GTA), dans lesquels les utilisateurs peuvent acquérir des maisons (homes), abris, planques, appartements, maisons, etc., qui servent souvent la narration du jeu. Outre l'incarnation de la réussite du personnage joué au travers de leurs représentations toujours plus vastes et luxueuses au fil des histoires jouées, ces « résidences » servent souvent de points de passage pour équiper l'avatar (en armes, en véhicules), pour sauvegarder sa progression ou encore pour marquer le début ou la fin d'une session de jeu. Si les possibilités d'aménagement sont souvent réduites, certains univers offrent la possibilité de sélectionner des objets (meubles, décorations) compilés dans des catalogues ou bibliothèques d'objets.

33 - les mondes virtuels dits sociaux (que l'on appelle parfois des tchats 3D), tels que IMVU, Twinity, Our World. Le principal usage de ce type d'univers est la rencontre entre membres par le biais de discussions informelles, d'événements divers, de jeux, etc. Les utilisateurs ont souvent la possibilité d'avoir un espace privé personnalisable (room, condo, etc.) et de produire, acheter, modifier et échanger des objets.

34 - les mondes virtuels de création de contenus, qui sont des univers produits majoritairement par leurs utilisateurs (spécifiquement dans le mode «créatif» de Minecraft par exemple). En 2010, Tom Boellstorff estimait que $99 \%$ des objets composant et habillant Second Life étaient l'œuvre de ses utilisateurs (Boellstorff, 2008, p. 97). Ainsi, par les environnements qu'ils créent, ces derniers contribuent activement à l'histoire et au déroulement des événements qui adviennent et qui façonnent à leur tour l'environnement virtuel (Nagy et Koles, 2016, p. 3).

Ces différentes catégories de mondes virtuels n'offrent pas les mêmes possibilités et les mêmes prises aux utilisateurs pour agir sur l'environnement qu'ils expérimentent et mettent en scène. Il est donc possible d'observer une pluralité de stratégies et de pratiques spatiales que les individus déploient et adoptent pour « faire avec l'espace », au sens où « l'être humain [...] doit en permanence accepter l'épreuve spatiale qui trouve sa 
source dans l'existence de la séparation, c'est-à-dire de l'impossible confusion des réalités sociales matérielles en un même point" (Lussault, 2007, p. 35). Car, même dans les mondes virtuels, l'avatar a une corporalité matérielle et psychique difficilement superposable avec d'autres avatars. De fait, certains utilisateurs font «avec» l'espace alors qu'ils n'ont pas les droits pour agir avec et sur lui. Ils trouvent des manières de faire «sans» ces droits, puisqu'ils ne peuvent pas faire sans l'espace. Nous appelons ces utilisateurs des "habitants-opportunistes». Il y a également ceux qui «ne font pas", alors qu'ils ont les droits, en ce sens qu'ils n'exploitent pas les prises qui s'offrent à eux ou que leur faible expérience sensible du monde les contraint d'avoir recours à des repères partagés, sans avoir la possibilité de faire émerger leurs propres prises. Ce sont les « habitants-bridés ». Ensuite, il y a ceux qui « font dans », c'est-à-dire ceux qui exploitent les prises qui leur sont offertes et qu'ils génèrent à l'intérieur de leur résidence. Nous qualifions ces utilisateurs d' ' habitants-aménageurs ». Enfin, les « habitants-bâtisseurs » sont ceux qui «font ", en ce sens qu'ils saisissent l'ensemble des prises pour agir autant sur le contenant (intérieur) que le conteneur (extérieur) de leur résidence.

Nous proposons de détailler plus précisément ces modalités d'action grâce aux quatre figures de l'habitant dans les mondes virtuels que nous venons d'introduire.

\section{Esquisse d'une typologie des habitants des mondes virtuels}

\section{L'habitant-opportuniste (« fait sans »)}

Dans les mondes virtuels, un abri n'existe que parce que des choix narratifs ou liés au gameplay l'exigent. À ce titre, dans le mode «survie » de Minecraft, il est nécessaire de se «planquer» (dans un abri, une grotte, etc.) chaque nuit pour se protéger des monstres. Dans les mondes virtuels de création de contenus et dans les mondes dits sociaux, pour lesquels la notion de «vie » n'existe pas (ou rarement), l'abri n'a pas d'utilité: «Une maison! Pourquoi une maison? Il ne pleut jamais ici :-). Et puis même, ça serait mon avatar qui serait trempé, pas moi ", déclarait no-name-457. Dans Second Life, de nombreux individus considèrent ainsi qu'il est inutile d'être propriétaire d'un espace, tant le nombre de lieux accessibles, voire vacants, est important : «Si tu veux être peinard, y'a plein d'endroits où tu peux aller, où tu peux rester, où y'a jamais personne. En plus, certains propriétaires sont cools et tu peux laisser quelques objets. Après c'est sûr que t'es pas chez toi », déclare Bronkozor.

Bien que le "squat» n'existe pas au sens strict du terme dans les mondes virtuels, nombreux sont ceux qui profitent des territoires laissés « ouverts " par les propriétaires ou administrateurs; ces derniers souhaitant par exemple partager ce bien avec la communauté, vendre des objets, organiser des événements, valoriser leurs productions, etc. (Lucas, 2013a).

Il est intéressant de constater qu'un utilisateur qui ne possède pas de lieu «à lui » peut prendre pour «habitude» de se connecter ou se déconnecter d'un même endroit, à l'image de ceux qui mettent en place des " conventions de phasage ", qui sont des phases en début de session durant lesquelles un joueur va prendre le temps de se remettre « dans la peau » de son avatar (Auray, 2003). C'est ce que fait Makoun, un avatar de Second Life que nous avons rencontré. Ce dernier occupe habituellement un espace de manière 
opportuniste au point que celui-ci est devenu son «point de chute ». Il déclare même considérer ce lieu " un peu comme le sien », bien qu'il n'ait aucune possibilité d'action sur lui. Il est donc incapable de faire plier ce lieu à son image. Malgré cela, il éprouve une certaine "attache » à ce lieu, dont il affectionne particulièrement l'ambiance. Alors qu'aucune trace, aucune empreinte, aucun stigmate n'est lisible après son passage, l'« habitant-opportuniste» peut éprouver un certain ancrage psychique pour un lieu, c'est-à-dire un " attachement ", parce qu'un lien s'est créé, qu'il est attaché à lui, affecté par lui (Latour, 2000 ; Hennion, 2010). Paradoxalement, alors qu'il ne possède pas le lieu qu'il occupe par la présence de son avatar, il peut être "possédé par lui ». Ainsi, par la répétition de l'ancrage corporel de leur avatar en un même lieu (habitude), certains individus développeront le sentiment de loger, voire d'habiter dans un lieu qu'ils ne possèdent pas, et au sein duquel ils ne peuvent pas se répandre. En un sens, l'habitantopportuniste est plus habité par le lieu qu'il ne l'habite. En revanche, cet ancrage corporel et affectif reste précaire, car soumis à l'accord d'un tiers qui peut choisir à tout moment de restreindre l'accès au lieu qu'il partage. D'une certaine manière, le sentiment d'habiter, voire l'« effet d'habiter », peut alors se dissiper rapidement.

\section{L'habitant-bridé («fait pas »)}

40 L'« habitant-bridé » possède un espace par « défaut ». C'est par exemple l'utilisateur de mondes dit sociaux qui va bénéficier d'un " espace à lui », mais qui ne va pas l'investir ou le personnaliser. Il ne va rien en faire, il ne « fait pas » avec l'espace, du moins avec cet espace. Ce peut également être l'utilisateur de Second Life qui, à la souscription d'un compte Premium, se voit attribuer une "résidence privée ", mais qui ne s'y rend et s'y répand que très peu. Les raisons de ce non-engagement dans l'aménagement et la personnalisation d'un logement peuvent être multiples : manque d'intérêt de la part de l'utilisateur, prises offertes trop restreintes en nombre ou en potentiel d'action, expérience trop limitée pour en faire émerger d'autres, manque de différenciation entre les logements des différents utilisateurs, etc. Dans Second Life, un avatar nous faisait part de son sentiment : «Sur le moment je me suis dit que ça allait être sympa. Mais c'est nul. Franchement. Déjà, quand j'arrive, je ne sais jamais laquelle c'est. [...] J'y pose des objets, des trucs comme ça, mais bon, je n'en fais rien d'essentiel », explique Mickytik. À l'image de ce témoignage, les seules traces de l'emprise d'un lieu par un habitant-bridé vont être la présence de quelques possessifs.

41 Ce type d'utilisateurs est bridé (par le système légal, technique, par son manque d'expérience, etc.), car s'il possède un lieu, en tant qu'il en est le propriétaire, il ne le possède pas en tant qu'objet ou bien approprié. Par conséquent, l'utilisateur n'est ni affecté, ni attaché, ni possédé à et par ce lieu. Dans ce cas, la dimension psychique de l'ancrage au lieu est faible et l'habitant-bridé apparaît, d'une certaine manière, «endetté » de ce bien dont il ne sait que faire. Néanmoins, malgré les limitations, les contraintes et le non-investissement de l'individu dans la relation avec sa propre résidence, celle-ci établit des seuils spatiaux, sociaux et symboliques, entre différents espaces (Segaud, 2010, p.130). Elle agit comme un opérateur de différenciation qui structure "l'espace de soi et des autres", qui dissocie le dehors du dedans, "ce qui m'appartient et ce qui appartient à autrui » (Averlant et Sabatier, 2008). En ce sens, l'habitat-centre de l'habitant-bridé constitue un ancrage géographique et structurel, qui lui permet d'être localisé et localisable dans le monde puisque son identité est liée à ce 
lieu par des références diverses (inscription dans un annuaire, coordonnées spatiales, URL, etc.).

\section{L'habitant-aménageur («fait dans »)}

42 À la différence de l'habitant-bridé, l'« habitant-aménageur » saisit les prises qui lui sont offertes et qu'il fait émerger pour agir sur l'environnement et confectionner un intérieur en modifiant par exemple son agencement et son habillage (meubles, tapisseries, objets décoratifs, etc.). L'habitant-aménageur n'a aucune prise pour agir sur l'extérieur de son logement, comme c'est le cas dans de nombreux mondes virtuels qui n'en proposent pas de représentation graphique (on accède à son logement via un bouton, un raccourci, etc.) ou qui se contentent d'une représentation symbolique, identique à tous les utilisateurs (un immeuble sur une carte symbolise le logement). L'habitant-aménageur fait plus qu'aménager un intérieur, il le confectionne et le climatise (Sloterdijk, 2006). Il produit un « univers d'expériences » (Musso, Coiffier et Lucas, 2014) qui va conditionner son rapport au monde. En cela, l'habitant-aménageur est un «designer d'atmosphère " (Desroches, 2011).

43 Cette production d'un intérieur contribue à l'appropriation de l'espace, au sentiment de le faire sien, et à la production d'un espace d'intimité dans lequel l'utilisateur peut s'isoler, se recueillir (Bachelard, 2010, p. 24) et exprimer son intériorité (Serfaty-Garzon, 2003, p. 67). Lieu de conventions de phasages, d'intimité ou encore de partage et d'hospitalité, puisqu'on peut y inviter ses amis, l'espace intérieur produit par l'habitantaménageur devient habitation. Celle-ci renforce sa relation au monde virtuel en affirmant la zone d'habitat-central de l'individu (au sein de celui-ci) et en participant, de fait, à renforcer les dimensions narrative, sociale et émotionnelle de son immersion.

\section{L'habitant-bâtisseur («fait »)}

44 Dans les mondes virtuels, un builder (que nous traduisons en français par «bâtisseur ») désigne un utilisateur qui génère du contenu. Suivant cette conception, l'habitantaménageur qui produit des objets peuplant l'intérieur de sa résidence est un builder. Néanmoins, au travers de cette figure, nous souhaitons nommer les utilisateurs qui modélisent et modèlent entièrement leur lieu de résidence, c'est-à-dire autant son contenant que son conteneur. Nous considérons qu'un utilisateur qui agit sur l'intérieur et l'extérieur de son habitat-centre renforce son « emprise » sur ce lieu, notamment parce que l'ancrage structurel qui peut le caractériser devient une trace architecturale reconnaissable et identifiable par tout le monde. De plus, l'extérieur modelé par l'utilisateur forme un couplage avec l'intérieur, qui se traduit par la cristallisation d'une enveloppe produisant une bulle expérientielle au sein de laquelle l'individu va produire un rapport spécifique au monde.

\section{Ancrages, traces et prises}

Les figures de l'habitant dans les mondes virtuels peuvent être distinguées selon trois notions qui caractérisent leurs expériences spatiales, donc sociales. D'abord, celle de "prise ", que nous avons déjà définie. Notons néanmoins que celles-ci peuvent être 
impossibles à générer (dans le cas de l'habitant-opportuniste), non saisies (l'habitantbridé) ou encore saisies et exploitées (habitant-aménageur et l'habitant-bâtisseur).

Ensuite, celle de "trace ». L'émergence d'une habitation dans un monde virtuel revêt la possibilité pour les utilisateurs de laisser une forme particulière de trace à l'intérieur de celui-ci :

J'aime bien les jeux vidéo, je suis un gros gamer, mais tout le monde se déplace dans le même environnement, ou presque. Une fois que le jeu est fini, ton passage n'a rien changé, car tu peux recommencer la partie à zéro. Quand tu joues, tu es simplement un joueur qui passe sans jamais laisser de traces. J'aime Second Life et je joue aussi beaucoup à Minecraft, car ce que tu fais reste, ça ne part pas en fumée (Nomaz).

Ce qui me plait dans Second Life, c'est que tu peux construire des objets qui restent, qui sont à toi, qui te correspondent. T'es pas juste un joueur qui défile dans un monde préconstruit. C'est toi qui construit le monde, qui build des trucs. Quand tu pars et que tu reviens, tu vois encore tes productions. Elles sont là, elles restent. Pour le moment on ne réalise pas, mais imagine dans 10, 20, 30 ans... on dira: ce monument, ça fait 30 ans qu'il est là, et 30 millions d'avatars l'ont visité. On visitera peut-être ces mondes comme on visite des grottes et des musées et on fera peutêtre des statues pour célébrer la mémoire de certains avatars (Charlibix).

Une fois de plus, le droit est décisif quant aux possibilités données aux utilisateurs de laisser des traces dans un monde virtuel. Par exemple, l'habitant-opportuniste ne peut pas laisser de traces, alors que le bâtisseur laissera, par le biais ses possessifs matérialisés dans l'habitation qu'il a produite, ainsi que par l'architecture qu'il lui a donnée, une trace identitaire forte. Les traces laissées par un individu sont directement dépendantes des prises que ce dernier saisit ou génère, en même temps qu'elles constituent des repères possibles pour l'action, soit des prises potentielles.

Enfin, celle de «l'ancrage ». Les homes, rooms, maisons, appartements, etc., représentent souvent des points de fixation dans l'expérience de l'utilisateur, c'est-à-dire des « stations » marquant des points d'arrêts dans le « mouvement » (Radkowski, 2002), c'està-dire dans l'organisation de leur relation au monde. Avoir un ancrage, c'est avoir une attache, un lien avec un lieu pour lequel on est localisé et localisable. L'attachement à un ancrage se définit dans une relation bilatérale entre un individu et un lieu, puisqu'on s'y attache autant qu'on y est attaché. Son intensité est donc variable selon les individus, et chacun traînera avec lui, dans les va-et-vient qui définissent l'élasticité de leur «l'habitat-milieu » (Radkowski, 2002), un « lestage » (Boullier, 2017) qui lui est propre. La dimension spatiale d'un ancrage peut être décrite au travers de la fréquentation habituelle d'un lieu par un avatar (ancrage corporel), par la possession d'un espace localisable (ancrage géographique), ou encore par celle d'un bien matériel (ancrage structurel). Sa dimension temporelle est définie par un contrat - ou son absence - qui lie un individu avec un lieu : dans le premier cas, elle est définie, car contractuelle, dans le second elle est précaire, car dépendante d'un tiers qui peut cesser à tout moment de tolérer la présence d'individus sur son territoire.

Le tableau ci-dessous synthétise les prises qu'ont les différents types d'habitant pour agir sur et avec l'espace, le type de traces qu'ils peuvent laisser dans le monde ainsi que la nature de leur ancrage par rapport à leur habitant-centre, ou résidence. Il souligne l'argument selon lequel l'habitant-aménageur et l'habitant-bâtisseur peuvent habiter un monde virtuel, puisque ce sont les seuls à pouvoir faire émerger une habitation à l'intérieur d'un tel environnement. Pour autant, nous avons montré que l'habitantopportuniste et l'habitant-bridé, même s'ils ne peuvent faire l'expérience respective que 
d'un ancrage psychique ou d'un logement, expérimentent et ressentent certains effets du processus de l'habiter. ${ }^{11}$

Figure 1

\begin{tabular}{|c|c|c|c|c|c|}
\hline & & \multicolumn{4}{|c|}{ Figures de l'habitant } \\
\hline & & Opportuniste & Bridé & Aménageur & Bâtisseur \\
\hline \multirow{2}{*}{ Prises } & $\begin{array}{ll}\text { sur } & \text { le } \\
\text { contenant } & \end{array}$ & aucune & $\begin{array}{l}\text { peu ou non } \\
\text { saisies }\end{array}$ & saisies & saisies \\
\hline & $\begin{array}{ll}\text { sur } & \text { le } \\
\text { conteneur } & \end{array}$ & aucune & aucune & aucune & saisies \\
\hline \multicolumn{2}{|c|}{ Type de traces } & aucune & possessif & $\begin{array}{l}\text { possessif } \\
\text { atmosphère }\end{array}$ & $\begin{array}{l}\text { possessif } \\
\text { atmosphère } \\
\text { architectural }\end{array}$ \\
\hline \multirow[t]{2}{*}{ Ancrage } & spatial & corporel & $\begin{array}{l}\text { corporel } \\
\text { géographique } \\
\text { structurel }\end{array}$ & $\begin{array}{l}\text { corporel } \\
\text { géographique } \\
\text { structurel }\end{array}$ & $\begin{array}{l}\text { corporel } \\
\text { géographique } \\
\text { structurel }\end{array}$ \\
\hline & temporel & précaire & contractuel & contractuel & contractuel \\
\hline \multicolumn{2}{|c|}{$\begin{array}{l}\text { Négociation du «faire } \\
\text { avec » l'espace }\end{array}$} & «fait sans » & «fait pas » & « fait dans » & «fait » \\
\hline \multicolumn{2}{|c|}{ Emergence } & $\begin{array}{l}\text { ancrage } \\
\text { psychique }\end{array}$ & logement & \multicolumn{2}{|l|}{ habitation } \\
\hline
\end{tabular}

Tableau synthétique des figures de l'habitant dans les mondes virtuels

\section{Conclusion}

Les quatre figures de l'habitant dans les mondes virtuels que nous avons présentées dévoilent différentes manières que les utilisateurs ont de « faire avec l'espace » (Lussault, 2007). Au-delà du désir d'accéder à un espace au sein d'un monde virtuel, de l'occuper, de le personnaliser et de se l'approprier, les pratiques des individus sont dépendantes des actions qui leur sont autorisées (par le système technique, ses possibles et son système de droit), suggérées (par les repères qu'ils détectent) et générées (par les prises saisies). Chaque figure illustre ainsi un «capital spatial » spécifique, c'est-à-dire un ensemble de « ressources qui permettent à l'acteur de faire avec l'espace [...], mais aussi de fabriquer sa propre spatialité dont l'espace sortira, peu ou beaucoup, modifié » (Lévy, 2015, p. 400).

51 Par le biais ces figures, nous avons également montré que le concept de l'habiter permet de décrire des pratiques spatiales, donc sociales, que des utilisateurs ont dans les mondes virtuels par le truchement de leur avatar. Notre démonstration suggère que tous les utilisateurs n'habitent pas les mondes virtuels, puisque certains n'ont pas, ou ne saisissent pas, la possibilité de tendre vers l'habiter. Souvent, ils ne peuvent pas affecter 
le monde de leur présence ou de leur action, et ne peuvent être affectés en retour. Pourtant, comme nous l'avons montré grâce à la figure de l'habitant-opportuniste, il est possible pour certains d'être attaché à un lieu et d'avoir «l'impression d'habiter ». En somme, si tous ne peuvent pas habiter un monde virtuel, d'aucuns peuvent faire l'expérience de certains effets de ce processus.

En outre, notre travail illustre le fait que les mondes virtuels sont des terrains d'observation privilégiés pour discuter certains concepts ; et en même temps démontrer qu'il n'est pas nécessaire de recourir à des néologismes pour caractériser des réalités qui ont lieu dans des environnements numériques.

La problématique de l'habiter dans les univers virtuels est une piste de recherche prometteuse au regard de l'évolution exponentielle des dispositifs qui permettent à des utilisateurs de se rencontrer dans des espaces en trois dimensions personnalisables ${ }^{12}$. En ce sens, notre travail mériterait d'être poursuivi selon plusieurs voies. Premièrement, en testant la pertinence des figures de l'habitant proposées grâce à une plus large diversité d'univers virtuels afin, notamment, d'éprouver l'articulation entre les notions de prise, de trace et d'ancrage selon un plus grand nombre de situations et de configurations vécues par des utilisateurs et leurs avatars. Ensuite, il semble intéressant de pouvoir approfondir le travail mené quant aux stratégies que les utilisateurs déploient pour réguler l'interspatialité entre les avatars, c'est-à-dire l'espace qui les relie et les sépare, au travers de la dyade avatar-habitat. Enfin, nous estimons que l'habiter dans les mondes virtuels doit être questionné au regard de l'hybridation qui unit ces univers avec notre pratique quotidienne du monde dans lequel notre être séjourne. Peut-être, alors, pourrons-nous faire l'hypothèse que les ancrages dont un individu fait l'épreuve à l'intérieur de ces univers contribuent à étendre l'« habitat-milieu » dont il fait l'expérience quotidienne.

\section{BIBLIOGRAPHIE}

ADAMS E. (2004), « Postmodernism and the Three Types of Immersion », Gamasutra. http:// www.designersnotebook.com/Columns/063_Postmodernism/063_postmodernism.htm

ARSENAULT D. (2006), « En eaux troubles : lumière sur l'immersion », Dire. La recherche à votre portée, vol. 16, $\mathrm{n}^{\circ} 1$, pp. 46-49.

AURAY N. (2003), » L'engagement des joueurs en ligne : ethnographie d'une sociabilité distanciée et restreinte ", Les Cahiers du numérique, vol. 4, nº 2, pp. 83-100.

AURAY N. (2005), « Sosies et avatars dans les jeux : entre écriture et image », in M. Durand, M. Gruau, J. Py et al. (dir.), L'image-sosie. L'original et son double, Actes du 1er colloque international Icône-Image, 8-10 Juillet 2004, Les Trois P. Obsidiane, pp. 95-108.

AVERLANT P. et SABATIER R. (2008), « Habiter, habitant, habitat », VST - Vie sociale et traitements, $n^{\circ}$ 97, pp. 26-32.

BACHELARD G. (2010 [1957]), La poétique de l'espace [1957], Paris, PUF. 
BEAUDE B. (2012), « Les jeux vidéo comme espaces de médiation ludique », in H. Ter Minassian, S. Rufat, S. Coavoux (dir.), Espaces et temps dans les jeux vidéo, Questions théoriques, pp. 28-52.

BERRY V. (2011), « Sociologies des MMORPG et profils de joueurs : pour une théorie sociale de l'activité (vidéo)ludique », Revue des Sciences Sociales, $\mathrm{n}^{\circ} 45$.

BERRY V. et BROUGERE G. (2002), « Play and Virtual Communities on Internet », $22^{\text {nd }}$ ICCP World Play Conference, Erfurt (RFA) 6-8 juin 2001, Actes du colloque, http://www.iccp-play.org/ documents/erfurt/berry-brougere.pdf

BESSY C. et CHATEAURAYNAUD F. (2014 [1995]), Experts et faussaires : pour une sociologie de la perception, Paris, Pétra, $2^{\mathrm{e}}$ édition revue et augmentée.

BOELLSTORFF T. (2008), Coming of Age of Second Life : An Anthropologist Explores the Virtually Human, Princeton University Press, First paperback printing.

BOUCHARDON S. (2009), Littérature numérique, le récit interactif, Paris, Lavoisier.

BOULLIER D. (2008), «Le Web immersif », Quaderni, nº6, pp. 67-80.

BOULLIER D. (2017), « Hotel California ou habitèle ? », conférence donnée le 25 juillet 2017 dans le cadre du colloque « Carte d'identités. L'espace au singulier », 22/29 juillet 2017, Cerisy-la-Salle.

CALLEJA G. (2007), « Digital Game Involvement. A conceptual model », Games and Culture, vol. 2, n - 3, pp. 236-260.

COOPER R. et DIBBELL J. (2007), Alter Ego : avatars and their Creators, Londres, Chris Boot.

DAMER B., GOLD S., MARCELA K., et REVI F. (1999), Inhabited Virtual Worlds in Cyberspace, in J.-C. Heudin (dir.) Virtual Worlds, Synthetic Universes, Digital Life, and complexity, Reading (Mass.) : Perseus books, pp. 127-152.

DESROCHES D. (2011), « L'homme comme designer d'atmosphère : Sloterdijk et la critique des milieux métaphysiques », Transverse, pp .39-52.

ERMI L. et MÄYRÄ F. (2005), « Fundamental Components of the Gameplay Experience : Analysing Immersion », Proceedings of DiGRA Conference : Changing Views, Worlds in Play. http:// citeseerx.ist.psu.edu/viewdoc/download?doi=10.1.1.103.6702\&rep=rep1\&type=pdf FOUILLE L. (2010), L'attachement automobile mis à l'épreuve : étude des dispositifs de détachement et de recomposition des mobilités, thèse de doctorat, Université de Rennes 2.

GENETTE G. (1972), Figures III, Paris, Le Seuil.

GENVO S. (2012), « Comprendre et développer le potentiel expressif », Hermès, La Revue, $\mathrm{n}$ ${ }^{\circ} 62$, pp. 127-133.

GENVO S. et BOUCHARDON S. (2009), " Méthodes d'évaluation en sémiotique du gameplay », LutinGameLab Rapport final ANR, Annexes scientifiques au rapport final, pp. 133-145. https:// halshs.archives-ouvertes.fr/halshs-00371331/file/annexes_scientifiques_Lutin_Game_Lab.pdf GEORGES F. (2010), Identités virtuelles. Les profils utilisateur du web 2.0, Questions Théoriques.

GIBSON J.J. (1986), The Ecological Approach to Visual Perception, Hillsdale (MI), Lawrence Erlbaum. GUELTON B. (2014), «Introduction. Figures de l'immersion », in B. Guelton (dir.), Les figures de l'immersion, Rennes, Presses Universitaires Rennes, pp. 9-22.

HEIDEGGER M. (2012 [1958]), Essais et conférences, Paris, Gallimard.

HENNION A. (2010), « Vous avez dit attachements ?... », in M. Akrich (dir.), Mélanges en l'honneur de Michel Callon, Paris, Presses de l'Ecole des Mines. 
JANKOVIC N. (2003), « Habiter l'Internet », in R. Drouhin (dir.), Habiter l'Internet, Rennes, Association des écoles supérieures d'art de Bretagne.

JOLIVEAU T. (2012), « Les lieux réels dans le jeu vidéo. Contribution à une approche géographique des espaces vidéoludiques ", in H.Ter Minassian, S. Rufat, S. Coavoux (dir.), Espaces et temps dans les jeux vidéo, Questions théoriques, pp. 148-181.

KAPLAN D. (2007), « Préface », in F. Beau (dir.), Culture d'Univers: Jeux en réseau, mondes virtuels, le nouvel âge de la société numérique, Limoges, FYP Editions, pp. 8-9.

KRUEGER M.W. (1983), Artificial Reality, Boston, Addison-Wesley.

LATOUR B. (2000), « Factures/fractures. De la notion de réseau à celle d'attachement », in A. Micoud et M. Peroni (dir.), Ce qui nous relie, La Tour d'Aigues, editions de l'Aube, pp. 189-208.

LEMIEUX C. (1995), « C. Bessy, F. Chateauraynaud, Experts et faussaires. Pour une sociologie de la perception », Politix, $n^{\circ} 31$, pp. 228-232. http://www.persee.fr/doc/

polix_0295-2319_1995_num_8_31_1927

LEROUX N. (2008), «Qu'est-ce qu'habiter ? Les enjeux de l'habiter pour la réinsertion », VST - Vie sociale et traitements, $\mathrm{n}^{\circ} 97$, pp. 14-25.

LESSIG, L. (1999), Code and Other Laws of Cyberspace, New York, Basic Books.

LEVY J. (2015), « Habiter Cheonggyecheon : l'exception ordinaire », Annales de géographie, Habiter : mots et regards croisés, $\mathrm{n}^{\circ}$ 704, pp. 391-405.

LUCAS J.-F. (2012), « Interactions et réalité mixte dans la ville hybride », in Z. Khaldoun (dir.), HyperUrbain 3 : Villes hybrides et enjeux de l'aménagement des urbanités numérique, Actes de colloque HyperUrbain.3, Europia Production, pp. 47-63.

LUCAS J.-F. (2013a), De l'immersion à l'habiter dans les mondes virtuels. Le cas des villes dans Second Life, thèse de doctorat, Université européenne de Bretagne, Rennes 2. http://tel.archives-ouvertes.fr/ tel-00949125

LUCAS J.F. (2013b), « Le projet Magic Ring : expérimentation d'une méthode de recueil de données quali-quantitatives dans Second Life », tic\&société, vol. 7, n² 2 http:// journals.openedition.org/ticetsociete/1561

LUCAS J.-F. et AMATO E.A. (2013), « Mondes, points de vue, personnages : l'avatar comme enveloppe pilotable », in E. A. Amato, E. Perény (dir.), Les avatars jouables des mondes numériques : Théories, terrains et témoignages de pratiques interactives, Paris, Lavoisier, pp. 109-133.

LUSSAULT M., (2007), « Habiter, du lieu au monde : Réflexions géographiques sur l'habitat humain ", in T. Paquot, M. Lussault, C. Younès (dir), Habiter, le propre de l'humain. Villes, territoires et philosophie, Paris, La Découverte, pp. 35-52.

MAUCO O. (2013), GTA IV, l'envers du rêve américain : Jeu vidéo et critique sociale, Questions théoriques.

MEADOW M.S. (2007), I, Avatar : The Culture and Consequences of Having a Second Life, New Riders. MURRAY J.H. (1997), Hamlet on the Holodeck: The Future of Narrative in Cyberspace, Cambridge, The MIT Press.

MUSSO P., COIFFIER S., et LUCAS J.-F. (2014), Innover avec et par les imaginaires, Paris, Editions Manucius. 
NAGY P. et KOLES B. (2016), «'I Create Therefore I Virtually Exist' : Digital Content Creation, Virtual Consumption, and Motivation in Second Life ", Journal of Virtual World Research, vol. 9, $\mathrm{n}$ $\circ 2$.

PAQUOT T., (2007), « Introduction. “Habitat”, “habitation”, “habiter”, précisions sur trois termes parents ", in T. Paquot, M. Lussault, C. Younès (dir), Habiter, le propre de l'humain. Villes, territoires et philosophie, Paris, La Découverte, pp. 7-16.

PEARCE C. (2009), Communities of Play. Emergent Cultures in Multiplayer Games and Virtual Worlds, The MIT Press.

PEREC G. (2000 [1974]), Espèces d'espaces, Paris, Galilée,.

PERRIAULT J. (2009), « Traces (numériques) personnelles, incertitudes et lien social », Hermès, La Revue, $\mathrm{n}^{\circ}$ 53, pp. 13-20.

QUEAU P. (1993), Le virtuel, vertus et vertiges, Paris, Champ Vallon.

RADKOWSKI de G.-H. (2002), Anthropologie de l'habiter : vers le nomadisme, Paris, PUF.

RHEINGOLD H. (1993), La réalité virtuelle, Paris, Dunod.

SANSOT P. (2004 [1996]), Poétique de la ville, Paris, Payot \& Rivages.

SAUVAGE A. (2007), « Raisons d'habiter. Pour une modélisation anthropologique », in T. Paquot, M. Lussault, C. Younès (dir), Habiter, le propre de l'humain. Villes, territoires et philosophie, Paris, La Découverte, pp. 69-88

SERFATY-GARZON P. (2003), Chez soi, les territoires de l'intimité, Paris, Armand Colin.

SEGAUD M. (2010), Anthropologie de l'espace: Habiter, fonder, distribuer, transformer, 2 édition, Paris, Armand Colin.

SERVAIS O. (2012), « Autour des funérailles dans World of Warcraft. Ethnographie entre religion et mondes virtuels ", in J.-P. Delville (dir.), Mutations des religions et identités religieuses, Paris, Desclée, pp. 231-252.

SLOTERDIJK P. (2006), Sphères III, Ecumes : Sphérologie plurielle, Paris, Hachette littératures.

TURKLE S. (1995), Life on the Screen: Identity in the Age of the Internet, New York, Simon and Schuster.

\section{NOTES}

1. Qui est accessible $24 \mathrm{~h} / 24,7 \mathrm{j} / 7$, qui évolue en l'absence d'utilisateurs connectés et qui cumule les actions de ces derniers.

2. A titre d'exemple, voir la revue «Presence» du M.I.T (http://www.mitpressjournals.org/loi/ pres) sur la notion de présence. Quant à l'intensité de l'expérience de l'utilisateur, voir Brown et Cairns (2004), entre autres, qui ont recours à cette notion pour définir différents degrés du processus immersif au sein des mondes virtuels.

3. Ces citations sont issues d'entretiens menés à l'intérieur des mondes virtuels, donc avec les avatars, entre 2009 et 2011, dans le cadre de notre thèse de doctorat. La méthodologie générale de ce travail s'apparente à une ethnographie telle que l'a défini Tom Boellstorff (2008), c'est-àdire que nous nous sommes uniquement intéressé aux usages et pratiques des individus à l'intérieur du monde virtuel, sans jamais questionner la vie des utilisateurs quand ils n'y sont pas connectés. Pour plus d'informations sur la méthodologie utilisée dans ce travail doctoral, se reporter à Lucas (2013a, 2013b). 
4. Les noms des avatars ont été modifiés.

5. En italique dans le texte.

6. Il serait intéressant de porter une attention plus spécifique aux manières dont les systèmes de droit (interdire, ou punir en cas d'actions de vandalisme) conditionnent les pratiques spatiales des individus dans les mondes virtuels, en tant qu'ils établissent des frontières et des seuils, donc qu'ils influent sur les relations de voisinage entre les avatars, c'est-à-dire sur la nature même de la coprésence en ligne.

7. « Squatter », Trésor de la Langue Française informatisé (TLFi) : http://stella.atilf.fr

8. A titre d'exemple, voir les actions listées par Georges Perec lorsqu'il évoque celle d'emménager (Perec, 1974, 2000, p. 71).

9. A ce propos, voir la notion de «méta-jeu » chez Berry et Brougère (2002).

10. Ces mondes sont couramment appelés des « sandboxes » («bacs à sable », en français), mais ce terme désigne également des solutions qui ne sont pas des mondes virtuels (les jeux de construction et de gestion de parc à thèmes sont couramment nommés comme tel). L'expression «monde virtuel de création de contenus » est donc plus appropriée, d'autant que certaines zones de ces environnements sont des sandboxes, d'autres non.

11. C'est pour cette raison que nous avons choisi d'utiliser le nom d'habitant comme un préfixe pour nommer nos quatre figures.

12. Voir par exemple « Spaces » (https://www.facebook.com/spaces) de Facebook, qui propose à ses utilisateurs équipés de casques de réalité virtuelle de se rencontrer, d'échanger et de créer et partager des contenus au sein de « scènes virtuelles » modélisées en trois dimensions.

\section{RÉSUMÉS}

Les utilisateurs et les avatars des mondes virtuels en ligne sont parfois qualifiés d'habitants, mais ce terme n'est presque jamais défini. L'objet de cet article est de caractériser ce qui fait qu'un habitant habite un monde virtuel. Nous considérons que l'immersion est une condition préalable à l'habiter dans un monde virtuel, puisque habiter implique de faire l'expérience d'un « intérieur ». Ensuite, grâce à des observations réalisées à l'intérieur de mondes virtuels ainsi qu'à une série d'entretiens issus de travaux préliminaires, nous décrivons les manières de «faire avec l'espace » des avatars grâce aux notions d'ancrage, de prise et de trace. L'habiter est alors appréhendé comme le couplage résultant de l'actualisation de ces trois notions, encapsulées dans les possibles d'un monde virtuel, par les actions d'un utilisateur. Ainsi, nous proposons quatre figures de l'habitant dans les mondes virtuels pour rendre compte de la diversité des manières d'y construire des expériences spatiales, donc sociales. Si tous les utilisateurs n'habitent pas un monde virtuel, notre grille heuristique permet d'en caractériser, au moins, certains aspects et effets.

Users and avatars of online virtual worlds are sometimes referred to as inhabitants, although this term is almost never defined. The purpose of this article is to characterize what makes that an inhabitant "inhabits" a virtual world. We consider that immersion is a prerequisite to inhabit a virtual world, since it implies experiencing an "interior". Then, thanks to observations carried out inside virtual worlds as well as a series of interviews resulting from preliminary work, we describe the ways of "doing with space" of the avatars thanks to the notions of anchor, catch and trace. The concept of "inhabit" is then apprehended as the coupling resulting from the updating 
of these three notions, encapsulated in a virtual world, by the actions of a user. Thus, we propose four figures of the inhabitant of virtual worlds to account for the diversity of ways of constructing spatial, and therefore social, experiences. At least, if not all users inhabit in a virtual world, our heuristic grid allows us to characterize some of its aspects and effects.

INDEX

Mots-clés : habitant, habiter, mondes virtuels, résidence, ancrage, prise, trace, immersion Keywords : inhabitant, inhabiting, dwelling, virtual worlds, residence, anchorage, trace, immersion

\section{AUTEUR}

\section{JEAN-FRANÇOIS LUCAS}

Laboratoire de sociologie urbaine (LaSUR), École polytechnique fédérale de Lausanne 